\title{
Ein Baum überlebt sein Schloss
}

\author{
Hilke Steinecke
}

\begin{abstract}
Since 2009 champion trees are listed by the German Dendrological Society. One of these trees is a 300 years old plane tree (Platanus $\mathrm{x}$ hispanica) growing near Unna. The tree and its history is described.
\end{abstract}

\section{Zusammenfassung}

Seit 2009 weist die Deutsche Dendrologische Gesellschaft Rekord-Bäume aus. Einer von ihnen ist eine 300 Jahre alte Platane (Platanus x hispanica) in der Nähe von Unna. Der Baum und seine Geschichte werden vorgestellt.

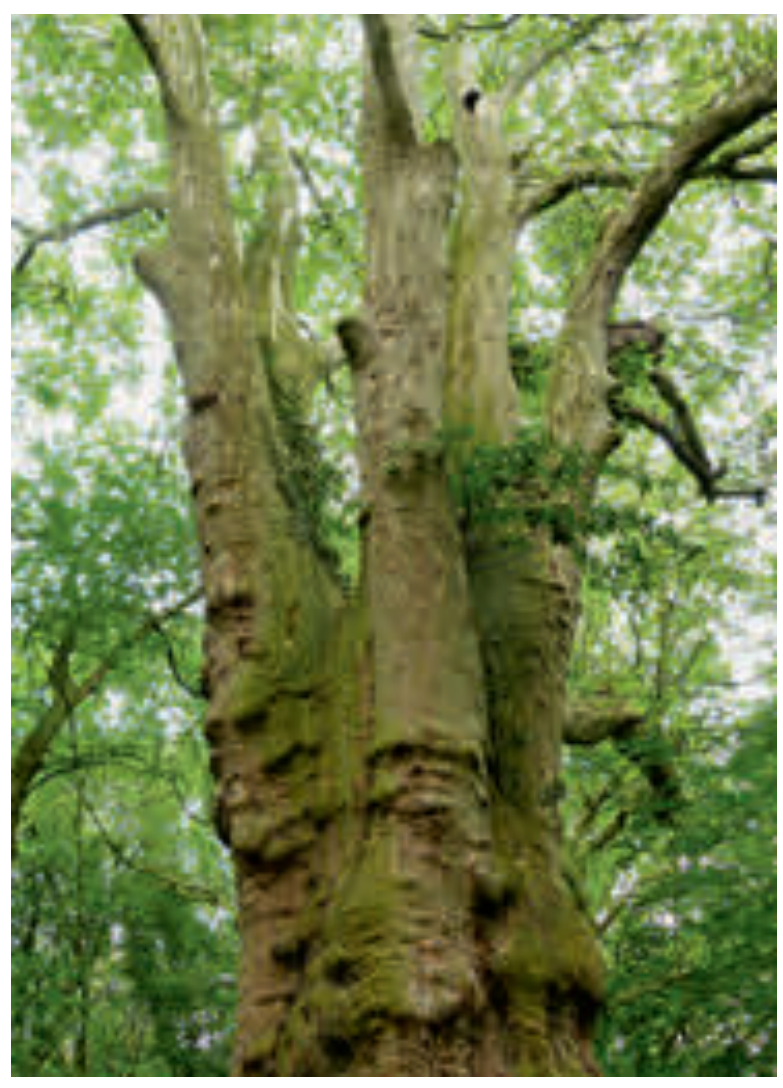

\section{Geschichte des Hauses Heyde}

In Westfalen (vor allem im Münsterland und in den angrenzenden Gebieten wie z.B. im Kreis Unna) gibt es viele attraktive Wasserschlösser und Güter. Am bekanntesten ist das prächtige Schloss Nordkirchen, das auch als Versailles Westfalens bezeichnet wird. Charakteristisch sind die Wassergräben (Gräften), die das Anwesen schützend umgeben. Nicht alle Schlösser und Güter haben die Zeit überlebt, einige sind verfallen oder wurden abgerissen, an manche erinnern nur noch Reste der ehemaligen Gräften oder ein alter Baumbestand. Eines dieser nicht mehr vorhandenen Anwesen ist das einstige Rittergut Haus Heyde. Es handelte sich um einen ehemaligen Adelssitz im westfälischen Dorf Uelzen, heute ein Stadtteil von Unna. Erstmals erwähnt wurde Haus Heyde (oder auch Heide) im Jahr 1343. Verschiedene Adelsfamilien waren in seinem Besitz, darunter die Familien vON TORCK, vON DER RECKE, vON Plettenberg und die berühmten von BodelSCHWINGHS.

Unter Christoph Friedrich Steffen von Plettenberg (1698 - 1777) erlebte Haus Heyde seine beste Zeit. Durch Veräußerungen seiner Besitztümer und Erlösen aus der Teilnahme am ersten Schlesischen Krieg (1740 - 1742) verfügte von Plettenberg über die finanziellen Mittel, im Jahr 1743 für 40722 Reichstaler das Gut zu kaufen. Er stattete den Landsitz üppig aus und pflanzte im Garten verschiedene Bäume. Sein Sohn Henrich Ludwig (1744-1799) baute das Haus Heyde im Barockstil zu einem Schlösschen um und legte einen Park an, aus dem noch heute einige Bäume erhalten sind. Durch Heirat gelangte Haus Heyde schließlich in den Besitz der Familie von BodelsCHWINGH, die es vier Generationen lang hielt, bis es 1927 an die Stadt Kamen verkauft wurde. Diese verpachtete es bis 1960 an verschiedene Landwirte. Von 1955 - 1965 war Haus Heyde

Abb. 1 (oben): Die alte, stattliche Platane von Haus Heyde.

Abb. 4 (Seite 89): Infotafel im ehemaligen Park von Schloss Heyde mit einer Abbildung des Südwestflügels des Schlosses um 1957. 
an den Jugendverband der Musikantengilde verpachtet, und es fanden dort regelmäßig Kammerkonzerte sowie Kinderfreizeitangebote und Zeltlager statt. Als die Stadt Kamen die Pachtverträge kündigte, entschied man sich 1966, das sich im schlechten Zustand befindende Haus Heyde abzureißen. Durch Flächentausch und Verkauf gelangte die Kernfläche des ehemaligen Rittergutes an die Stadt Unna. Im Zuge des Abbruches wurde auch ein Teil der Gräften zugeschüttet.

\section{Im alten Schlosspark}

Im Gebiet rund um das ehemalige Haus Heyde befindet sich heute ein Wäldchen, das aus dem verwilderten Park hervorgegangen ist. Einige bemerkenswerte alte Bäume erinnern aber noch an die „gute alte Zeit“. Als Naturdenkmal ausgezeichnet sind zwei Hybrid-Platanen (Platanus acerifolia $=P$. $\mathrm{x}$ hispanica), zwei Rotbuchen $(F a-$ gus sylvatica), eine Stiel-Eiche (Quercus robur) und eine Rosskastanie (Aesculus hippocastanum). Zudem gedeihen hier mehrere echte
Schwarz-Pappeln (Populus nigra ssp. nigra). Auch unter den Tieren gibt es hier einige Besonderheiten. Hier wurden beispielsweise acht verschiedene Fledermausarten nachgewiesen, darunter der Kleine Abendsegler. Aufgrund des Artenreichtums wurde die Uelzener Heide (Gesamtfläche: 190 ha), dazu auch das Gelände des Hauses Heyde, 1997 unter Naturschutz gestellt.

\section{Die alte Platane, ein Rekordbaum}

Dem Besucher fällt beim Durchqueren des Wäldchens eine der beiden riesigen mit einem Informationsschild versehene Platanen auf. Diese Platane ist 250-300 Jahre alt und wurde vermutlich von Christoph Friedrich voN Plettenberg gepflanzt. Sie gehört somit zu den besonders alten Vertretern der Hybrid-Platane, die erst um 1650 durch Kreuzung aus der Amerikanischen (P. occidentalis) und der Morgenländischen Platane ( $P$. orientalis) entstanden ist. Der Baum hat vier Hauptstämme, in $1,30 \mathrm{~m}$ Höhe hat er einen Umfang von $712 \mathrm{~cm}$, ist $42 \mathrm{~m}$ hoch und weist einen geschätzten

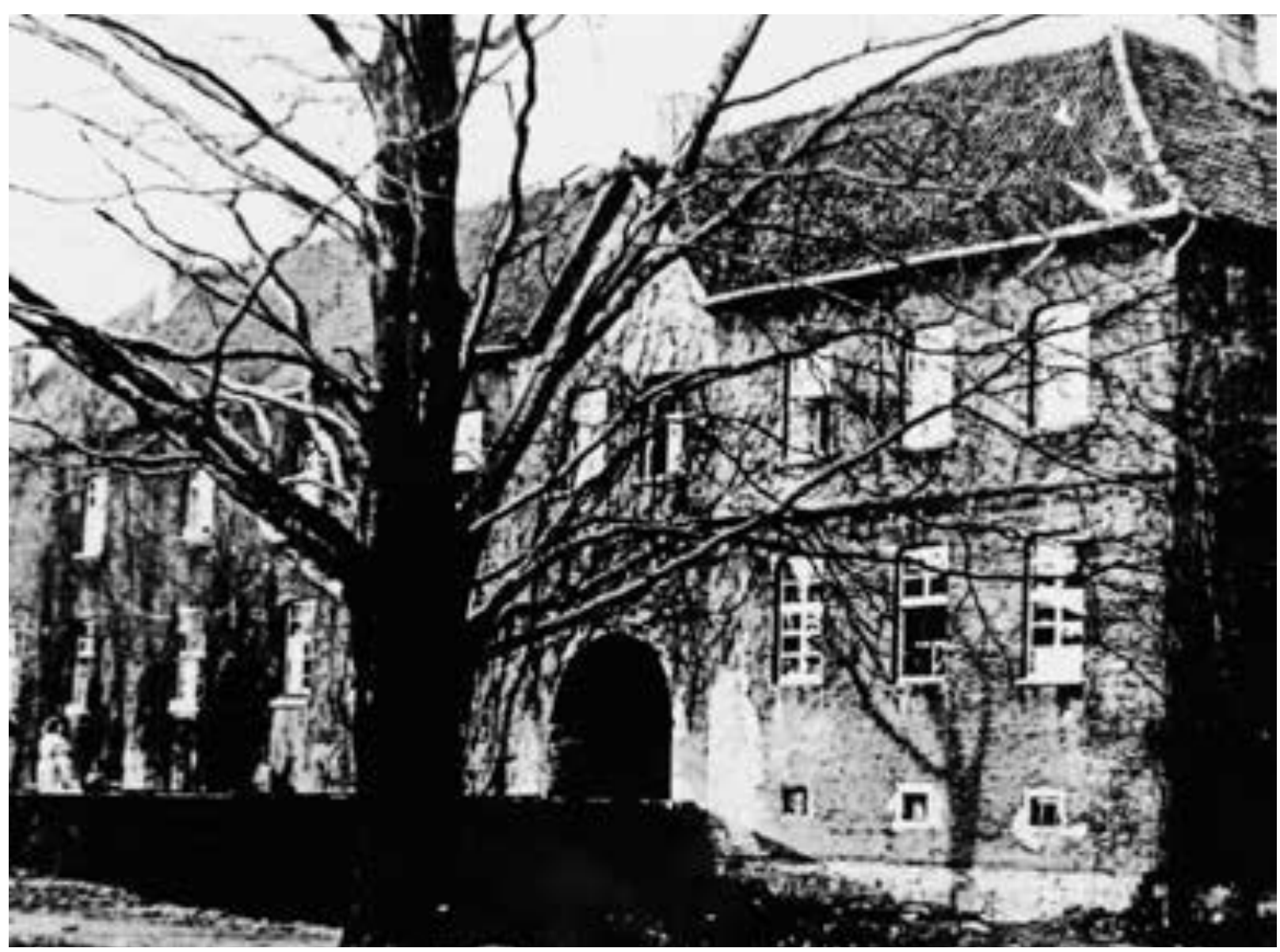




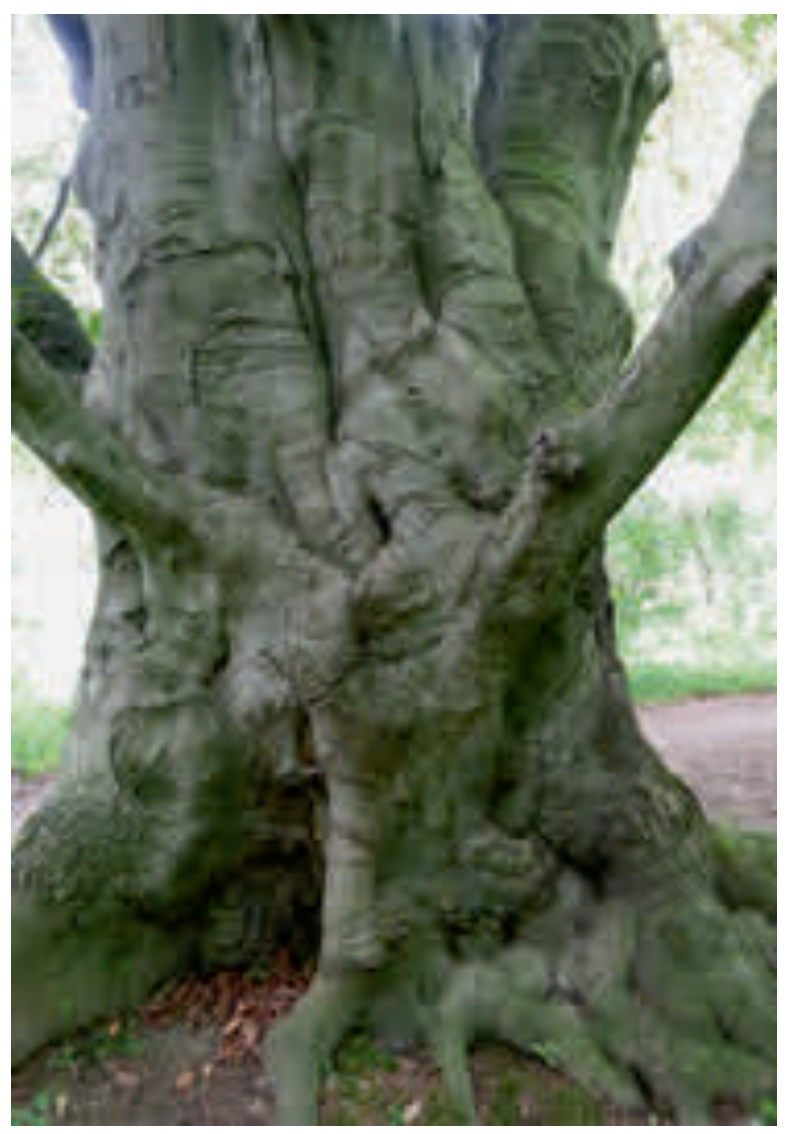

Abb. 3 (oben): In direkter Nachbarschaft zur Platane steht diese alte Buche mit verwachsenem Stamm.

Abb. 2 (unten): Der dicke Fuß der Platane.
Kronendurchmesser von $32 \mathrm{~m}$ auf. Auffällig sind seine tropfenförmigen Auswölbungen am Stamm.

Die Platane wurde am 13. August 2013 zum Champion Tree gekürt. Es handelt sich um die dickste Hybrid-Platane in Westfalen, möglicherweise auch von Nordrhein-Westfalen, bundesweit steht sie auf Platz 9. Rekord- oder Champion-Bäume werden von der Deutschen Dendrologischen Gesellschaft (DDG) und der Gesellschaft Deutsches Arboretum (GDA) seit 2009 ausgewiesen. Champion Trees in Deutschland sind jeweils die stärksten Bäume einer Art oder Sorte in einem Bundesland. In Nordrhein-Westfalen tragen gut 200 Bäume den Titel Champion Tree, in Hessen sind es rund 130 Rekord-Bäume.

\section{Internetseiten}

http://www.monumentaltrees.com/de/deu/ nordrheinwestfalen/unna/9044_hausheyde/ http://www.heimatverein-muehlhausen-uelzen.de http://de.wikipedia.org/wiki/Haus_Heyde http://www.championtrees.de/ was-ist-ein-champion-tree.html http://www.kreis-unna.de/fileadmin/user_upload/ Daten_und_Fakten___Statistik/Natur_und_Umwelt/X_ Naturschutzgebiete.pdf

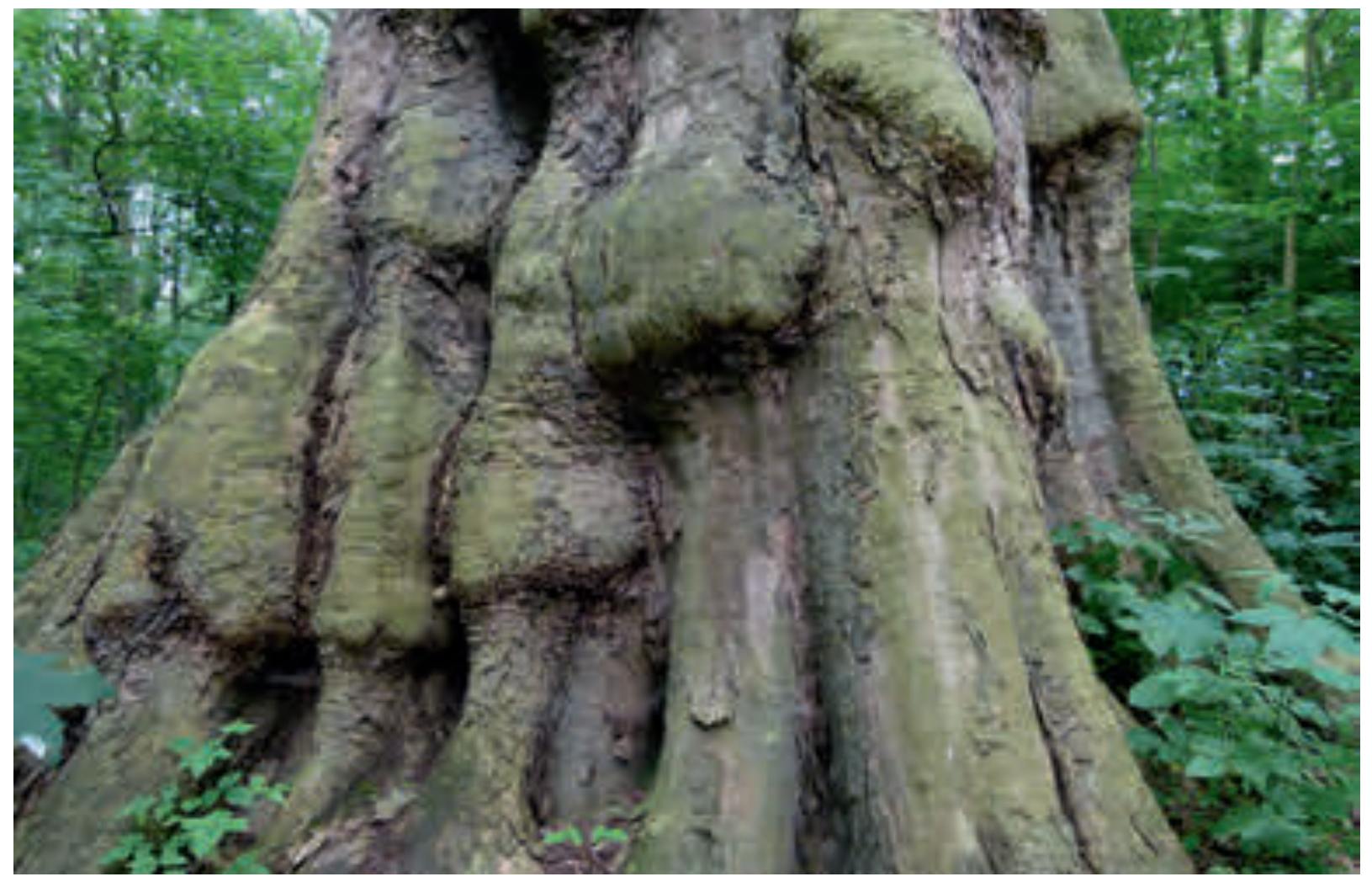

